\title{
A Method for Solving Reliability of Route Travel Time
}

\author{
Hongwei Ma, Gan Yi, Jianmin Qi $\mathbb{D}$, and Zhenjie Zhang $(\mathbb{D}$ \\ Shanghai DianJi University, Shanghai 201306, China \\ Correspondence should be addressed to Zhenjie Zhang; zhangzj12345678@sina.com
}

Received 10 March 2020; Accepted 3 April 2020; Published 27 April 2020

Academic Editor: Xinguang Zhang

Copyright (C) 2020 Hongwei Ma et al. This is an open access article distributed under the Creative Commons Attribution License, which permits unrestricted use, distribution, and reproduction in any medium, provided the original work is properly cited.

In order to obtain an effective method for solving route travel time reliability, this paper proposes an effective new method to calculate travel time reliability using an independent link travel time function. Based on several months of historical data, the results show that the Edgeworth expansion can better reflect travel time distribution law. In addition, travel time reliability can be calculated more conveniently by combining an approximate discretization algorithm and an empirical distribution function.

\section{Introduction}

Urban development has made traffic congestion increasingly severe, resulting in a high demand for a reliable transportation system. However, there are many stochastic factors that affect the systems, such as traffic congestion and road maintenance. These factors highlight the importance of a reliable transportation network. At present, the research on traffic network reliability mainly includes connectivity, travel time, and road network capacity. Travel time reliability is particularly important: it allows travelers to measure travel efficiency and perceive travel quality. The value of time is increasing and people are demanding shorter travel times. To achieve this, more attention must be paid to the volatility and reliability of travel time. The study of travel time reliability is of great significance for evaluating road network operations and improving travel quality.

Asakura and Kashiwadani proposed the concept of travel time reliability in 1991 [1]. Subsequently, scholars in different countries continue to develop this concept using time index, time delay index, and other indicators. In general, it is acceptable to use the probability measure function to calculate travel time reliability. Emam and Al-Deek estimated travel time reliability using data comprising the number and speed of vehicles provided by a coil detector on a highway in Orlando, Florida. They then assessed the goodness of fit using Weibull, exponential, lognormal, and Gaussian distri- butions. The results showed that the lognormal distribution was optimal for estimating reliability [2]. Wang et al. noted that travel behavior was impacted by travel time, travel time reliability, and monetary cost. Using these factors, she established the biobjective user equilibrium model of travel time reliability [3]. Bhouri and Aron studied factors that impacted travel reliability, such as hypersensitive reactions (e.g., tachycardia) on highways in France [4]. Hojatia et al. identified nonrecurring congestion using sudden time and location. The tobit model, which is based on extra buffer time, studies the impact of traffic accidents on travel time [5]. Hou and Tan established models for highway networks based on the Monte Carlo simulation to calculate the postearthquake connectivity reliability [6]. Among others, Tao and Zhang considered travel time reliability for multimodal transportation networks composed of commuters and cars [7]. Pan et al. addressed the dynamic networks to determine and control the risk of stochastic travel times. Then, they used optimization theory to develop algorithms to solve the problem [8].

The literature review revealed that most of the current research on travel time reliability determines the distribution function for travel time and establishes the reliability model. The function uses road and travel time statistics to determine the travel time distribution law. Travel time reliability is mainly used in analytical and simulation methods. Using historical data from a highway in Shanghai, this paper explores a new analytical method to solve for travel time reliability. 


\section{The Concept of Travel Time Reliability}

Travel time reliability is defined as the probability of completing a trip during traffic within a specified travel time. The basic equation is

$$
R(t)=P\left(T \leq t_{0}\right)
$$

In the equation above, $R(t)$ is the travel time reliability within designated sections, routes, or OD (Origin and Destination) pairs in a certain period of time; $T$ is the travel time within them; and $t_{0}$ is the specified travel time threshold. If the probability density function for the travel time of sections, routes, and OD pairs is $f(t)$, and the travel time distribution function is $F\left(t_{0}\right)$, then (1)

$$
R(t)=P\left(t \leq t_{0}\right)=\int_{0}^{t_{0}} f(t) d t=F\left(t_{0}\right) .
$$

\section{Solving for Route Travel Time Reliability}

Assume that a route $L$ consists of two sections $\left(a_{1}\right.$ and $\left.a_{2}\right)$ in series, the travel times of sections $a_{1}$ and $a_{2}$ are $X_{1}$ and $X_{2}$, respectively, and the joint density function of $X_{1}$ and $X_{2}$ is $f\left(x_{1}, x_{2}\right)$. The travel time of route $L$ is $Y$. The probability distribution function of $Y=x_{1}+x_{2}$ should be obtained first. The travel time reliability is then calculated using (2).

By definition, the distribution function of travel time $Y=x_{1}+x_{2}$ is

$$
F_{Y}(Y \leq y)=P\left(X_{1}+X_{2} \leq y\right)=\iint_{D} f\left(x_{1}, x_{2}\right) d x_{1} d x_{2}
$$

when $X_{1}$ and $X_{2}$ are independent, assuming that the edge density of $f\left(x_{1}, x_{2}\right)$, with respect to $X_{1}$ and $X_{2}$, is $f_{x_{1}}\left(x_{1}\right)$ and $f_{x_{2}}\left(x_{2}\right)$. Then, the probability density function of route travel time $\left(Y=x_{1}+x_{2}\right)$ becomes

$$
f_{Y}(y)=\int_{-\infty}^{\infty} f_{x_{1}}\left(y-x_{2}\right) f_{x_{2}}\left(x_{2}\right) d x_{2}
$$

or

$$
f_{Y}(y)=\int_{-\infty}^{\infty} f_{x_{1}}\left(x_{1}\right) f_{x_{2}}\left(y-x_{1}\right) d x_{1} .
$$

The distribution function is

$$
F_{Y}(y)=\int_{-\infty}^{y}\left[\int_{-\infty}^{\infty} f_{x_{1}}\left(x_{1}\right) f_{x_{2}}\left(y-x_{1}\right) d x_{1} d z\right.
$$

or

$$
F_{Y}(y)=\int_{-\infty}^{y} \int_{-\infty}^{\infty} f_{x_{1}}\left(y-x_{2}\right) f_{x_{2}}\left(x_{2}\right) d x_{2} d z
$$

3.1. Discrete Approximation Using an Empirical Distribution Function. If the travel time distribution function, $F(t)$, and the probability density function, $f(t)$, cannot be expressed

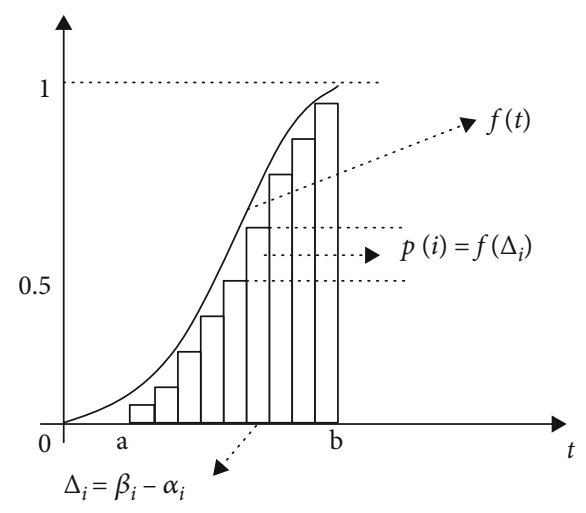

Figure 1: Discretization of empirical distribution of time travel.

in a formula, they can be approximated using empirical distribution. We can use the empirical distribution of travel time to calculate reliability. The travel time of continuous variables is treated as discrete variables.

As shown in Figure 1, if the time interval of study is $[a, b], t \in[a, b]$, the time interval $[a, b]$ is divided into two subareas: $[a, b]=\cup_{1}^{m}\left[\alpha_{i}, \beta_{i}\right]$ and $\Delta_{i}=\left[\alpha_{i}, \beta_{i}\right]$. The probability of travel time in $\left[\alpha_{i}, \beta_{i}\right]$ is recorded as $p(i)$, then $p(i)=$ $p\left(\alpha_{i} \leq t \leq \beta_{i}\right)=F\left(\beta_{i}\right)-F\left(\alpha_{i}\right)$. The travel time reliability of continuous variables can be approximated by a series of discrete values:

$$
R(t)=P\left(t \leq t_{0}\right)=\int_{0}^{t_{0}} f(t) d t \approx \sum_{i=1}^{m} p(i) .
$$

The travel time probability distribution of the two sections can be discretized using (8). Equation $P\left(x_{1}=i, x_{2}=j\right)$ calculates the probability of travel times $X_{1}$ and $X_{2}$ being less than $i$ and $X_{2}$ are independent,

$$
P\left(x_{1}=i, x_{2}=j\right)=P\left(x_{1}=i\right) \times P\left(x_{2}=j\right) .
$$

Then, the probability of route travel time, $r$, is

$$
P(Y=r)=\sum_{i=1}^{r} P\left(x_{1}=i, x_{2}=r-i\right)
$$

The reliability of route travel time not exceeding $t$ is

$$
\begin{aligned}
R(Y \leq t)= & P(Y \leq t)=\sum_{r=1}^{t} \sum_{i=0}^{r} P\left(x_{1}=i, x_{2}=r-i\right) \\
& \cdot \sum_{r=1}^{m} \sum_{i=0}^{r} P\left(x_{1}=i\right) P\left(x_{2}=r-i\right) .
\end{aligned}
$$

\subsection{Edgeworth Asymptotic Expansion Series Approximation} Using Density Function. Since the urban traffic system is complex, the law of travel time distribution is becoming less and less obvious. If the distribution of travel time cannot be expressed by known functions, we can use series expansion to approximate the distribution function and calculate travel time reliability. The Edgeworth expansion series 
approximates the characteristic equation of the distribution function using $F(t)$. The density function, $f(t)$, is another known distribution extension of the characteristic equation. The normal distribution is commonly selected and then passed through the inverse transformation of Fourier's equation. This obtains characteristic equation $F(t)$. To understand the main process, refer to references [9-11].

The Edgeworth expansion for probability density function was used to calculate travel time reliability. If the random variable travel time is $t$, the mean value is $\mu$, and the variance is $\sigma^{2}$, and if $y=(t-u) / \sigma$, then the probability density function is

$$
\begin{aligned}
p(y)= & \varphi(y)\left(1+\frac{\kappa_{3}}{3 !} H_{3}(y)+\frac{\kappa_{4}}{4 !} H_{4}(y)\right. \\
& \left.+\frac{10 \kappa_{3}^{2}}{6 !} H_{6}(y)+\frac{\kappa_{5}}{5 !} H_{5}(y)+\frac{35 \kappa_{3} \kappa_{4}}{7 !} H_{7}(y)+\cdots\right)
\end{aligned}
$$

In this equation, $\phi(y)$ is the standard normal distribution density function. The Hermite orthogonal polynomial, $H_{i}(y), H_{0}(y)=1, H_{1}(y)=y, H_{2}(y)=y^{2}-1, H_{3}(y)=y^{3}-3 y$, $H_{4}(y)=y^{4}-6 y^{2}+3 \cdots$;

$$
\begin{aligned}
H_{n}(y) & =(-1)^{n} e^{-y^{2} / 2} \frac{d^{n}}{d y^{n}} e^{y^{2} / 2} \\
& =y^{n} \sum_{k=1}^{n / 2}(-1)^{k}(2 k-1) ! ! C_{n}^{2 k} y^{n-2 k}, \\
& =\mu_{1}, \\
\kappa_{2} & =\mu_{2}-\mu_{1}^{2}, \\
\kappa_{3} & =\mu_{3}-3 \mu_{1} \mu_{2}+2 \mu_{1}^{3}, \\
\kappa_{4} & =\mu_{4}-3 \mu_{2}^{2}-4 \mu_{1} \mu_{3}+12 \mu_{1}^{2} \mu_{2}-6 \mu_{1}^{4}, \cdots .
\end{aligned}
$$

$u_{k}$ is the $k$-order moment estimator that can be calculated using actual observations.

Taking the four items ahead, (12) can be simplified to

$$
p(y)=\varphi(y)\left[1+\frac{s}{3 !}\left(y^{3}-3 y\right)\right]
$$

In this equation, $\phi(y)$ is the standard normal distribution probability density function and $s$ is the deviation of travel time. The corresponding distribution function is

$$
F(y)=\left[\varphi(y)-\frac{s}{3 !} \phi(y)\left(y^{2}-1\right)\right]
$$

In practice, the convergence of the Edgeworth expansion of probability density function is often neglected. The numerical experiments show that it can fulfill accuracy requirements. Specific details can be found in references $[10,11]$.
In (12), $\kappa_{i}$ and $\mu_{i}$ are constants. Equation (12) can be simplified to

$$
p(y)=\varphi(y)\left(\sum_{i=0}^{\infty} a_{i} y^{i}\right) .
$$

After the travel time probability density of the road section is obtained using equation (13), the route travel time reliability can be calculated using discrete approximation (Section 3.1) if the section travel time are independent. If the asymptotic expression of the travel time density function of the two sections is

$$
\begin{aligned}
& p\left(x_{1}\right)=\varphi\left(x_{1}\right)\left(\sum_{i=0}^{\infty} a_{i} x_{1}{ }^{i}\right) \\
& p\left(x_{2}\right)=\varphi\left(x_{2}\right)\left(\sum_{i=0}^{\infty} b_{i} x_{2}{ }^{i}\right) .
\end{aligned}
$$

Then, the probability density function of $y=x_{1}+x_{2}$ is

$$
\begin{aligned}
f_{Y}(y) & =\int_{-\infty}^{\infty} p_{x_{1}}\left(y-x_{2}\right) p_{x_{2}}\left(x_{2}\right) d x_{2} \\
& =\int_{-\infty}^{\infty} \varphi\left(y-x_{2}\right)\left(\sum_{i=0}^{\infty} a_{i}\left(y-x_{2}\right)^{i}\right) \varphi\left(x_{2}\right)\left(\sum_{i=0}^{\infty} b_{i} x_{2}{ }^{i}\right) d x_{2}, \\
F(Y) & =\int_{-\infty}^{y} \int_{-\infty}^{\infty} \varphi\left(z-x_{2}\right)\left(\sum_{i=0}^{\infty} a_{i}\left(z-x_{2}\right)^{i}\right) \varphi\left(x_{2}\right)\left(\sum_{i=0}^{\infty} b_{i} x_{2}{ }^{i}\right) d x_{2} d z .
\end{aligned}
$$

Equation (21) can be used to calculate the reliability of path travel time.

\section{Empirical Research}

Existing literature has revealed the diversity and uncertainty of travel time

distribution. The current distribution function is still unable to describe many cases. Empirical research was conducted using the travel time distribution of the Shanghai Outer Ring Expressway at typical peak hour (8:00-9:30 and 17:00-18:30) from May 1st to July 31st, 2015. As shown in Figure 2, the Edgeworth series curve approximation (blue line) for the density function of the on-ramps from Wuzhong Road to Caobao Road is compared with their respective histograms. During rush hour, the saturation flow is close to or already at maximum, and the travel time fluctuation range is large; compared to normal distribution, there is a certain degree of deviation marked by $s_{1}$ and $s_{2}$. The peak values are at $s_{1}=1.07$ and $s_{2}=1.93$ for morning travel times. The evening rush hour curve deviates to the left.

Compared with normal distribution, the Edgeworth expansion better reflects the distribution law of travel time. By calculating the absolute error between expected probability of the normal distribution, the expected probability of Edgeworth expansion, and the actual frequency of travel 

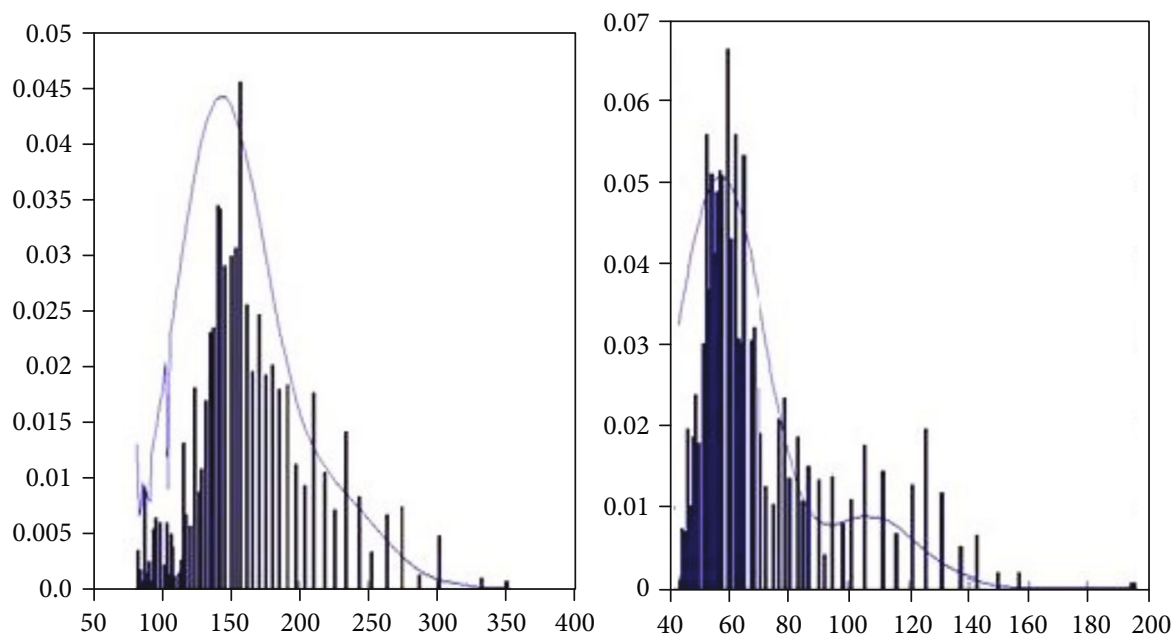

FIgURE 2: Comparing the Edgeworth series travel time probability density function and histograms.

TABLE 1: Error analysis of travel time estimation in early peak section.

\begin{tabular}{lcc}
\hline Category & $\begin{array}{c}\text { Normal } \\
\text { distribution }\end{array}$ & $\begin{array}{c}\text { Edgeworth expansion } \\
\text { distribution }\end{array}$ \\
\hline Maximum absolute error & 0.0709 & 0.0646 \\
Average absolute error & 0.0169 & 0.0138 \\
Sum of squares for error & 0.0138 & 0.0099 \\
\hline
\end{tabular}

TABLE 2: Error analysis of travel time estimation in late peak section.

\begin{tabular}{lcc}
\hline Category & $\begin{array}{c}\text { Normal } \\
\text { distribution }\end{array}$ & $\begin{array}{c}\text { Edgeworth expansion } \\
\text { distribution }\end{array}$ \\
\hline Maximum absolute error & 0.2615 & 0.184 \\
Average absolute error & 0.0475 & 0.0313 \\
Sum of squares of error & 0.0906 & 0.0438 \\
\hline
\end{tabular}

time, to compare the accuracy of travel time reliability, the differences are described using the maximum absolute error, the mean absolute error, and the sum of squares for error. The results are shown in Tables 1 and 2. It can be seen that the maximum absolute error value of travel time estimated by the Edgeworth expansion is less than the normal distribution, and the error between the estimated value of the Edgeworth expansion and the actual frequency is small. The accuracy of travel time distribution fitted by the Edgeworth expansion is higher than that of normal distribution, and the asymptotic distribution of travel time based on the Edgeworth expansion.

Using the distribution of section travel time, (21) can be used to calculate the reliability of travel time.

\section{Conclusion}

Travel time reliability is of great significance when analyzing the reliability of urban traffic networks. A new method for calculating travel time reliability was designed under the condition that the route travel times were independent. Based on several months of historical data, the results show that the Edgeworth expansion series can better reflect the distribution law of travel time. In addition, travel time reliability can be calculated more conveniently by combining an approximate discretization algorithm and an empirical distribution function. However, this method requires statistical traffic data to determine the empirical distribution function. The accuracy of the obtained results is closely related to the division of time interval. The accuracy and expanded forms of the Edgeworth series approximation for distribution functions are closely related. However, the quantitative relationship between its error and expansion needs to be studied further.

\section{Data Availability}

No data were used to support this study.

\section{Conflicts of Interest}

The authors declare that they have no conflict of interest.

\section{Acknowledgments}

This work was supported by "the Humanity and Social Science Youth Foundation of Ministry of Education of China (Grant No. 18YJC630120)" and "the National Natural Science Foundation of China (Grant No. 61273042)”.

\section{References}

[1] Y. Asakura and M. Kashiwadani, "Road network reliability caused by daily fluctuation of traffic flow," in Proceedings of the 19th PTRC Summer Annual Meeting, pp. 73-84, Brighton, 1991.

[2] E. B. Emam and H. Al-Deek, "Using real-life dual-loop dector data to develop new methodology for estimating freeway travel time reliability," in The 85th Annual meeting Transportation Research Board, pp. 125-134, Washington DC, 2007. 
[3] J. Y. T. Wang, M. Ehrgott, and A. Chen, "A bi-objective user equilibrium model of travel time reliability in a road network," Transportation Research Part B: Methodological, vol. 66, no. 8, pp. 4-15, 2014.

[4] N. Bhouri and M. Aron, "Isolating different factors affecting travel time reliability in an observational before/after study," Transportation Research Procedia, vol. 3, pp. 329-338, 2014.

[5] A. T. Hojati, L. Ferreira, S. Washington, P. Charles, and A. Shobeirinejad, "Modelling the impact of traffic incidents on travel time reliability," Transportation Research Part C: Emerging Technologies, vol. 65, no. 4, pp. 49-60, 2016.

[6] H. O. U. Li-wen and T. A. N. Jia-mei, "Estimating travel time reliability in urban transportation using Gram-Charlier distribution," Chinese Journal of Management Science, vol. 17, no. 6, pp. 139-146, 2009.

[7] T. Junyong and Z. Yong, "Time reliability analysis of multimodal transportation networks," Journal of Transportation Systems Engineering and Information Technology, vol. 15, no. 2, pp. 216-222, 2015.

[8] P. A. N. Yiyong, M. A. Jianxiao, and S. U. N. Lu, "Optimal path in dynamic network with random link travel times based on reliability," Journal of Jilin University (Engineering and Technology Edition), vol. 46, no. 3, pp. 412-417, 2016.

[9] H. Cramer, "On the history of certain expansions used in mathematical statistics," Biomerika, vol. 59, pp. 205-207, 1972.

[10] W. Feller, An introduction to probability theory and its applications, vol. 11, John Wiley, New York, 1966.

[11] M. G. Kendall and A. Stuart, The advanced theory of statistics, vol. 1, no. 4, 1977, Griffin, London, 1977. 